\title{
Sustainable Development of Tensioned Fabric Green Structure in the Form of Enneper
}

\author{
H. M. Yee, K. K. Choong, and M. N. Abdul Hadi
}

\begin{abstract}
Tensioned fabric structure in the form of Enneper minimal surface can be considered as a sustainable development for the green environment and technology, it also can be used to support the effectiveness used of energy and the structure. Sustainable development of tensioned fabric green structure in the form of Enneper minimal surface has been studied. The combination of shape and internal forces for the purpose of stiffness and strength is an important feature of fabric surface. For this purpose, form-finding using nonlinear analysis method has been carried out for Enneper minimal surface models with variables $u=v=0.6$ and $u=v=1.0$. Pattern of prestress in the resulting fabric surface has been studied. Enneper minimal surface models with variables $u=v=0.6$ and $u=v=1.0$ with fabric type A provides an alternative choice for practicing engineers to consider the tensioned fabric green structure in the form of Enneper minimal surface applied in the construction industry. It is expected to reduce the construction cost, shorten the construction time and improve the construction quality.
\end{abstract}

Index Terms-Tensioned fabric structure, minimal surface, form-finding.

\section{INTRODUCTION}

A tensioned fabric structure (TFS) also known as tensioned membrane structure is a structure where very thin doubly-curved coated fabric in tension is used as the main structural element. Its have been employed throughout recorded history as in tents. Reference [1] has stated that the materials used for membranes generally consist of a woven fabric coated with a polymeric resin. The two most commonly used membrane types are poly tetra fluoro ethylene (PTFE) coated plain weave glass-fibre fabrics and PVC coated plain weave nylon or polyester fabrics. A fabric is coated when a weather tight structure required. In that case, the fabric consists of three layers - one layer of woven yarns and two layers of coating materials. The coating protects the fabric from UV radiation degradation, rainwater and atmospheric moisture.

Reference [1] has mentioned that fabric is made of woven yarns as shown in Fig. 1. Warp and fill yarns that are twisted together. The yarns are weaved in such a way that threads are perpendicular to one another and they are alternately passing over and under each other. Long straight yarns are called warp yarns and the direction parallel to the warp yarns are called warp direction; whereas perpendicular yarns are called

Manuscript received May 5, 2014; revised August 25, 2014.

H. M. Yee and M. N. Abdul Hadiis are with the Faculty of Civil Engineering, Universiti Teknologi MARA, 13500 Permatang Pauh, Pulau Pinang, Malaysia (e-mail: yhooimin@yahoo.com, nasayz@yahoo.com).

K. K. Choong is with the School of Civil Engineering, USM, Engineering Campus, 14300 Nibong Tebal, Seberang Perai Selatan, P. Pinang, Malaysia (e-mail: cekkc@eng.usm.my). fill yarns and they are weaved alternately over and under the warp yarns. The direction that is parallel to the fill yarns are called fill direction. There are 3 different types of fabric based on classification by Japanese Association of Membrane Structure such as Type A membrane (PTFSE coated glass fibres fabric), Type B membrane (PVC coated glass fibres fabric) and Type $\mathrm{C}$ membrane (PVC coated polyester fabric). The durability of fabric type $\mathrm{A}$ in the form of Enneper is 20-30 years with excellent fire resistance and good dirt resistance also. While durability of fabric type B and $\mathrm{C}$ in the form of Enneper is 10 years with fair fire resistance and not good dirt resistance.
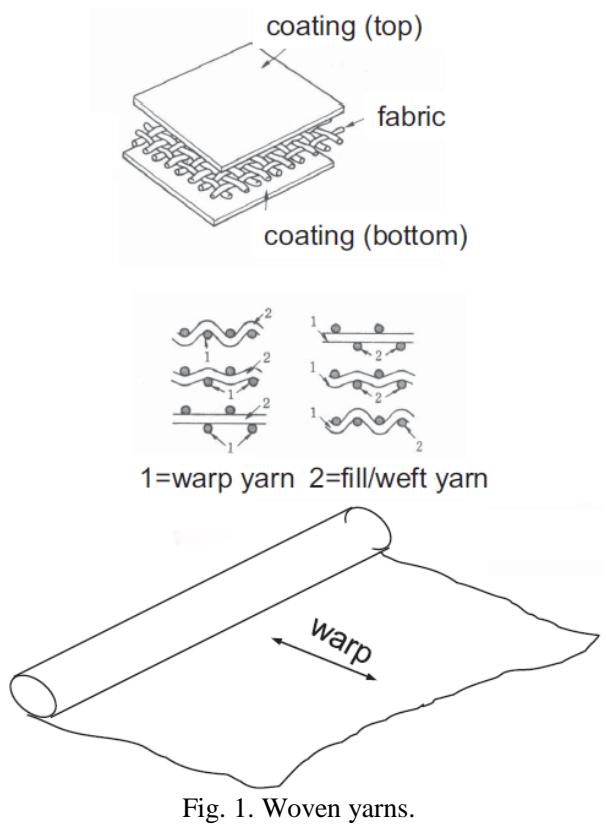

Reference [1] has stated that strength to weight ratio is one of the biggest advantage of tensioned fabric structures which can save materials, flexible, resistance to tears and high shear strength. Plain weave fabrics consist 2D layout of the undulated warp and weft threads. For example, polyvinyl chloride (PVC) coated polyester fabrics have tensile strength for warp and weft of $115 \mathrm{kN} / \mathrm{m}$ and $102 \mathrm{kN} / \mathrm{m}$, respectively. Weight of fabrics is Tensioned fabric structures with a high strength and lightweight can also provide a great variety of surface form. Light penetrates with natural light and artificial light provides an ambience of great appeal to humans. In day time, the light transmission is typically $5-20 \%$, it is sufficient to eliminate or greatly reduce the need for artificial lighting. Heat is reduced with little artificial lighting. Absorption of solar energy is typically $4-17 \%$. Tensioned fabric structures have minimal site interruptions since the fabric panels used for erection of TFS are manufactured in factories and TFS has low maintenance compared to glass. 
Reference [2] has stated that in recent years, the tensile surface structure business has grown considerably and is predicted to grow further. Such structures are becoming bigger and more sophisticated. Most tension structures are designed to have a uniform prestress in their fabrics. In this condition, there is no shear stress in the fabric. This is also the condition which minimizes the fabric surface area for a given set of initial conditions. Reference [3] presented an extension of the force density method called the natural force density method for the initial shape finding of cable and membrane structures, which led to the solution of a system of linear equations. With reference to a Helicoid soap film surface, the minimal surface associated with the prescribed boundary is obtained. Reference [4] presented numerical methods to simulate soap film experiments as well as how they could be integrated among themselves and with structural optimization. Reference [5] presented a study of minimum energy forms of prestressed cable nets and membranes in numerical form-finding and soap film models. Reference [6] used soap films to produce shapes of tension fabric structures. Soap film models effective means of finding shapes of an actual structure. Until today research on various topics of soap film continues across the country. Tensioned fabric structures are normally designed to be in the form of equal tensioned surface. Minimal surface such as classical Enneper minimal surface variation have been studied as possible choice of surface form for TFS by [1].

Reference [1] has stated that TFS is highly suited to be used for realizing surfaces of complex or new forms. Understanding of the possible initial equilibrium shapes to be obtained will provide alternative shapes for designers to consider. Before the shapes can be considered for structural application, their behaviour under load must be properly studied. For the case of tensioned fabric structures, the first step in any structural analysis is the form-finding. References [7]-[10] have carried out form-finding using nonlinear analysis method.

Form finding is to determine the initial equilibrium shape under pre-stress pattern and boundary condition. Reference [1] has proposed nonlinear analysis method for form-finding of tensioned fabric structures. References [1] and [11] have carried out form-finding using nonlinear analysis method in the form of Enneper minimal surface. Applicability of the computational strategies proposed has been verified by form-finding carried out models of tensioned fabric structures in the one of the form of Enneper minimal surface. Convergence was able to achieve in form-finding of Enneper TFS models. When $u=v \leq 0.87$, the converged shape is found to be similar to the mathematical shape. For Enneper TFS model ( $u=v \geq 0.88$ ), the shape obtained after form-finding are found to be different from the form of Enneper surface defined by mathematical shape equation. In this study, only form-finding using nonlinear analysis method proposed by [1] on Enneper minimal surface with variables $u$ and $v=0.6$ and 1.0 has been carried out in order to provides an alternative choice for structural engineer to consider the tensioned fabric green structure in the form of Enneper minimal surface so that able to improve green infrastructure, provision of economic gains, sustainability and green technology initiative for all the countries.

\section{GENERATION OF ENNEPER Minimal SURFACE IN TENSIONED FABRIC GREEN STRUCTURE}
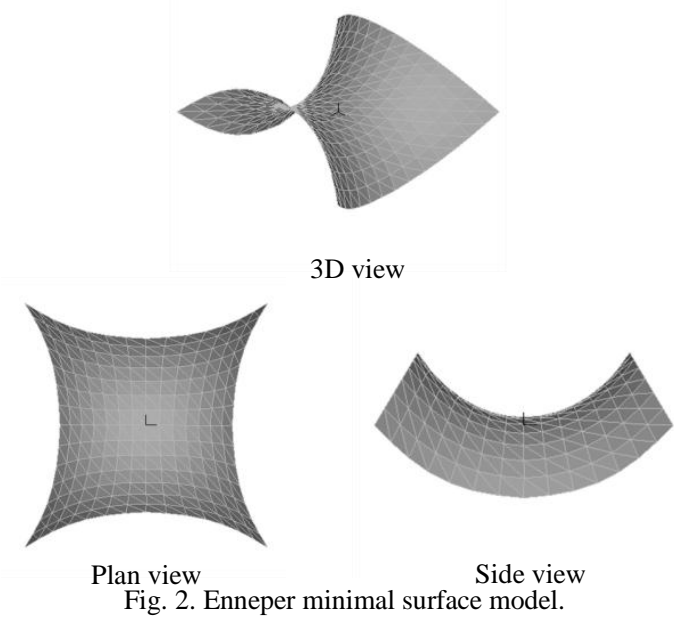

Fig. 2 shows Enneper minimal surface. The boundary of Enneper minimal surface in Fig. 2 can be obtained by using Eq. (1) [12].

$$
\begin{gathered}
X=u-\frac{u^{3}}{3}+u v^{2}, \\
Y=-v+\frac{v^{3}}{3}-v u^{2}, \\
Z=u^{2}-v^{2}
\end{gathered}
$$

for $u$ and $v=$ variables

For this study, the software [13] has been used for the purpose of model generation. Aspect of modeling of surface of Enneper minimal surface and form as well pre-stress pattern of the resulting TFS through form-finding using nonlinear analysis method are studied. The procedure adopted is based on the work by [1] and [14]. 3-node plane stress element has been used as element to model the surface of TFS. All $x, y$ and $z$ translation of nodes lying along the boundary edge of the Enneper minimal surface have been restrained. The member pretension in warp and fill direction, is $2000 \mathrm{~N} / \mathrm{m}$, respectively. The shear stress is zero.

\section{COMPUTATIONAL ANALYSIS FOR ENNEPER MiNIMAL SURFACE}

Form-finding using nonlinear analysis method proposed by [1] has been used in this study involved in the two phases, Phase I and Phase II. Two phases of analysis are involved in the procedures of form-finding based on stress analysis stage in one cycle. During Phase I, artificial tensioned fabric properties, namely elastic modulus $E$ with very small values relative to the actual $E$ value, are used. Both warp and fill tensioned fabric stresses, $\sigma_{\mathrm{W}}$ and $\sigma_{\mathrm{F}}$ are kept constant. In Phase II, the actual tensioned fabric properties values are used. Resulting warp and fill stresses are checked at the end of the analysis against prescribed tensioned fabric stresses. Iterative calculation has to be carried out in order to achieve convergence. The convergence of form-finding criteria adopted is that the total warp and fill stress deviation should be $<0.01$. The resultant shape at the end of iterative step has been considered to be in the state of initial equilibrium under 
the prescribed warp and fill stresses and boundary condition if difference between the obtained and the prescribed tensioned fabric stresses relative to the prescribed stress is negligibly small. Such checking of difference in the obtained and prescribed stresses have been presented in the form of convergent curve of variation of total stress deviation in warp and fill direction versus stress analysis stage.

In this study, Enneper minimal surface models with variables $u=v=0.6$ and $u=v=1.0$ have been presented. Total number of nodes and elements used in the Models are 225 and 392, respectively. Fig. 3 shows the initial equilibrium shape for Enneper minimal surface, $u=v=0.6$. Fig. 3 shows the convergence of the Enneper minimal surface, $u=v=0.6$. The convergence curve in Fig. 4 shows the total warp and fills deviation $<0.01$. The Enneper with the variables $u=v=0.6$ has a good agreement with the mathematically defined surface in Eq. (1) as shown in Fig. 5.

Fig. 6 shows the initial equilibrium shape for Enneper minimal surface, $u=v=1.0$. Fig. 7 shows the convergence of the Enneper minimal surface, $u=v=1.0$. The convergence curve in Fig. 7 shows the total warp and fills deviation $<0.01$.

\section{A. Enneper Minimal Surface, $u=v=0.6$}

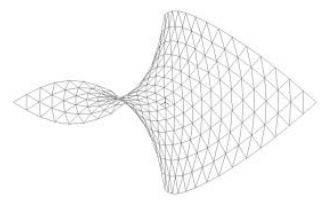

$3 \mathrm{D}$ view

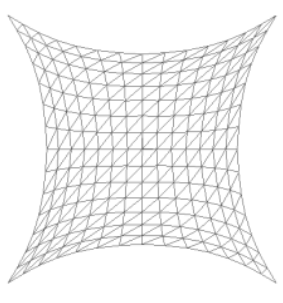

Plan view

Fig. 3. Different views of Enneper minimal surface, $u=v=0.6$.

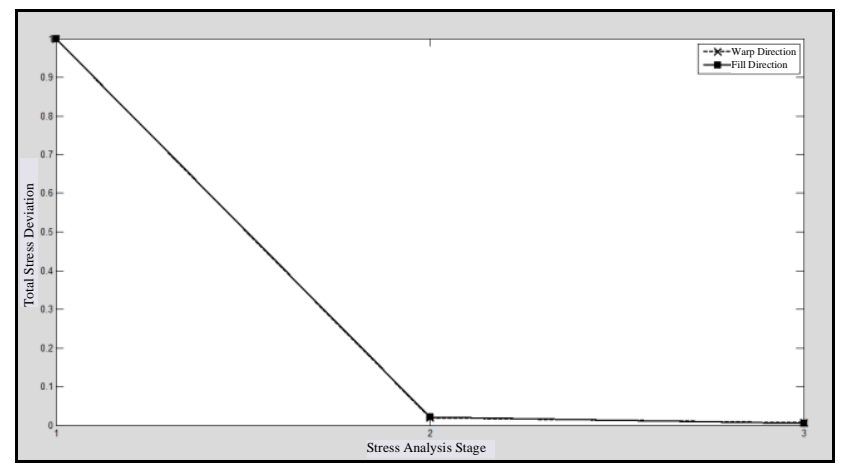

Fig. 4. Total warp and fills deviation for Enneper minimal surface $u=v=0.6$.

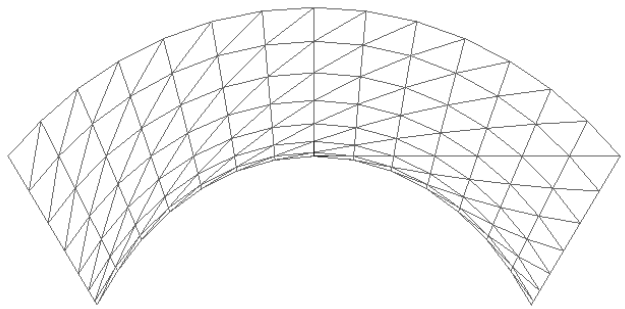

Fig. 5. Mathematical defined Enneper minimal surface, $u=v=0.6$.

\section{B. Enneper Minimal Surface, $u=v=1.0$}

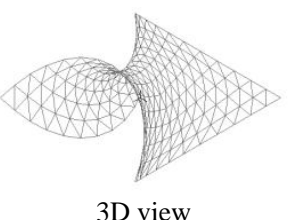

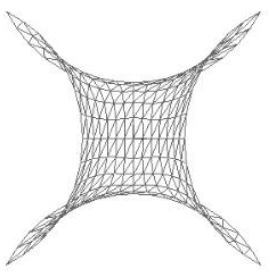

Plan view

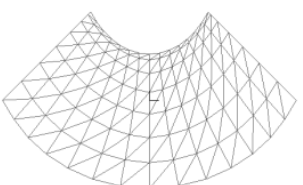

Side view
Fig. 6. Different views of Enneper minimal surface, $u=v=1.0$.

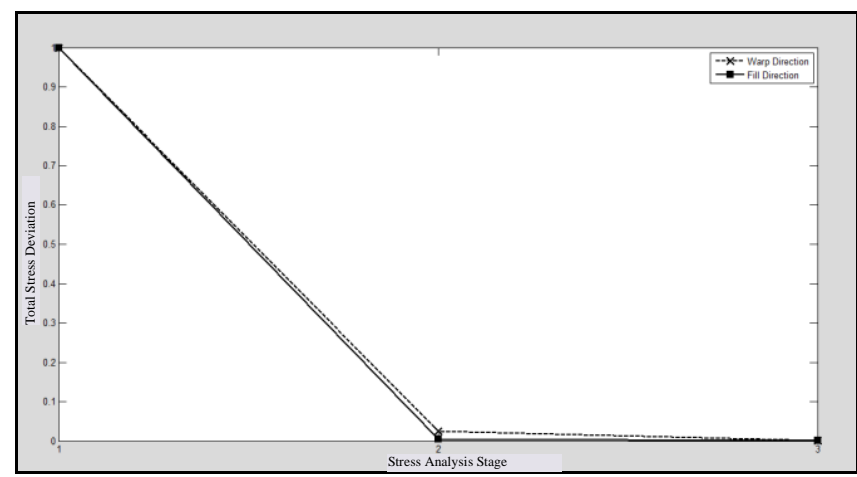

Fig. 7. Total warp and fills deviation for Enneper minimal surface $u=v=1.0$.

\section{CONCLUSION}

Form-finding with surface in the form of Enneper tensioned fabric green structure with variables $u=v=0.6$ and $u=v=1.0$ has been carried out successfully using the procedure adopted which is based on nonlinear analysis method. The results from this study show that tensioned fabric green structure in the form of Enneper minimal surface with fabric type A is a structurally viable surface form to be considered by engineer for sustainable development. Therefore, the proposed study TFS in the form of Enneper with variables $u=v=0.6$ and $u=v=1.0$ can save materials, provision of economic gains and reduce the environment impact by giving natural diffuse light with reduced heat load.

\section{REFERENCES}

[1] H. M. Yee, "A computational strategy for form-finding of tensioned fabric structure using nonlinear analysis method," Ph.D. dissertation, School of Civil Engineering, Universiti Sains Malaysia, Pulau Pinang, Malaysia, 2011.

[2] W. Xu, J. Ye, and J. Shan, Structural Engineering and Mechanics, vol. 31, pp. 349-365.

[3] M. O. Pauletti and P. M. Pimenta, "Shape finding of membrane structures by the natural force density method," in Proc. the Sixth International Conference on Computation of Shell and Spatial Structures IASS-IACM, USA, 2008, pp. 31-34.

[4] K. U. Bletzinger, R. Wüchner, F. Daoud, and N. Camprubi, "Computational methods for form-finding and optimization of shells and membranes," Computer Methods in Applied Mechanics and Engineering, vol. 194, no. 4, pp. 3438-3452, 2005.

[5] W. J. Lewis and P. D. Gosling, "Stable minimal surfaces in form-finding of lightweight tension structures," International Journal of Space Structures, vol. 8, no. 1, pp. 149-166, 1993.

[6] M. J. Hayati, "Form-finding of tension structures using soap film," Final year project, School of Civil Engineering, Universiti Sains Malaysia, Pulau Pinang, Malaysia, 2002. 
[7] H. M. Yee, J. Y. Kim, and M. S. Noor, "Tensioned fabric structures in oval form," Applied Mechanics and Materials, vol. 405-408, pp. 1008-1011, 2013.

[8] M. S. Noor, H. M. Yee, K. K. Choong, and H. Hamid, "Tensioned membrane structures in the form of egg shape," Applied Mechanics and Materials, vol. 405-408, pp. 989-992, 2013.

[9] H. M. Yee and K. K. Choong, "Form-finding of tensioned fabric structure in the shape of möbius strip," Iranica Journal of Energy and Environment, vol. 4, no. 3, pp. 247-253, 2013.

[10] H. M. Yee and M. A. Samsudin, "Development and investigation of the moebius strip in tensioned membrane structures," WSEAS Transactions on Environment and Development, vol. 10, pp. 145-149, 2014.

[11] H. M. Yee, K. K. Choong, and J. Y. Kim, "Form-finding analysis of tensioned fabric structures using nonlinear analysis method," Advanced Materials Research, vol. 243-249, pp. 1429-1434, 2011.

[12] A. Gray, Modern Differential Geometry of Curves and Surfaces with Mathematica, CRC Press LLC: United States of America, 1999, pp. 938-949.

[13] ADINA, System 8.1. R\&D Inc., 2003.

[14] H. M. Yee and K. K. Choong, "Proposed algorithm for warp direction checking in tensioned fabric structures," International Journal of Scientific Research in Knowledge, vol.1, no. 1, pp. 13-19, 2013.

Hooi Min Yee was born in Sungai Petani, Kedah, Malaysia, who graduated with a doctorate degree in structural engineering in 2011, master of science degree in engineering in 2007 and bachelor of engineering (honours) in civi engineering in 2006 from the Universiti Sains Malaysia, Pulau Pinang,
Malaysia.

During her PhD study, she worked as a fellow at Universiti Sains Malayisa. After graduation, she worked as an assistant professor at Faculty of Civil Engineering, Universiti Teknologi MARA, Pulau Pinang, Malaysia. Dr. Yee is a member of Board of Engineers Malaysia, Institution of Engineers Malaysia, Construction Industry Development Board Malaysia and Concrete Society of Malaysia. Dr. Yee has obtained 6 awards in Invention, Innovation and Research Design Platform and has published 37 up-to-date publications.

Kok Keong Choong graduated with a doctorate degree in architectural engineering from The University of Tokyo in 1994. After graduation, he worked as a postdoctoral research scholar under the late Professor Yasuhiko Hangai at Institute of Industrial Science, The University of Tokyo, Japan. Dr. Choong later joined Taiyo Kogyo Corporation as a research engineer at its Center for Space Structure Research in Osaka, Japan in from 1995 to 1999. In1996-1997, Dr. Choong was a postdoctoral research scholar sponsored by DAAD (German Academic Exchange Service) at University of Stuttgart where he worked with Prof. Ekkehard Ramm. Since 2000, Dr. Choong is an academic staff at the School of Civil Engineering, Universiti Sains Malaysia, Penang, Malaysia. At Universiti Sains Malaysia, Dr. Choong is involved in the teaching of undergraduate and postgraduate courses. He also conducted research in the area of computational analysis of shell spatial structures and bio-mimicry.

Mohd Nasir Abdul Hadi was born in Kuala Berang, Terengganu, Malaysia, who received bachelor of engineering (honours) in civil engineering in 2014 from the Universiti Teknologi MARA, Pulau Pinang, Malaysia and graduated with a diploma in civil engineering, Pahang, Malaysia. 